\title{
On the Maximum Estrada Index of 3-Uniform Linear Hypertrees
}

\author{
Faxu Li, ${ }^{1,2}$ Liang Wei, ${ }^{3}$ Jinde Cao, ${ }^{4,5}$ Feng $\mathrm{Hu}^{1,2}$ and Haixing $\mathrm{Zhao}^{2}$ \\ ${ }^{1}$ School of Computer Science, Shaanxi Normal University, Xi'an 710062, China \\ ${ }^{2}$ College of Computer, Qinghai Normal University, Xining 810008, China \\ ${ }^{3}$ Department of Mathematics, Qinghai Normal University, Xining 810008, China \\ ${ }^{4}$ Department of Mathematics, Southeast University, Nanjing 210096, China \\ ${ }^{5}$ Department of Mathematics, Faculty of Science, King Abdulaziz University, Jeddah 21589, Saudi Arabia
}

Correspondence should be addressed to Haixing Zhao; h.x.zhao@163.com

Received 3 July 2014; Accepted 12 August 2014; Published 28 August 2014

Academic Editor: Jianlong Qiu

Copyright (C) 2014 Faxu Li et al. This is an open access article distributed under the Creative Commons Attribution License, which permits unrestricted use, distribution, and reproduction in any medium, provided the original work is properly cited.

For a simple hypergraph $H$ on $n$ vertices, its Estrada index is defined as $E E(H)=\sum_{i=1}^{n} e^{\lambda_{i}}$, where $\lambda_{1}, \lambda_{2}, \ldots, \lambda_{n}$ are the eigenvalues of its adjacency matrix. In this paper, we determine the unique 3-uniform linear hypertree with the maximum Estrada index.

\section{Introduction}

Let $G=(V, E)$ be a simple graph, and let $n$ and $m$ be the number of vertices and the number of edges of $G$, respectively. The characteristic polynomial of a graph $G$ is written as $P(G, \lambda)=$ $\operatorname{det}(\lambda I-A(G))$, where $A(G)$ is the adjacency matrix of $G$. The eigenvalues of $G$ are the eigenvalues of its adjacency matrix $A(G)$, which are denoted by $\lambda_{1}, \lambda_{2}, \ldots, \lambda_{n}$. A graphspectrum-based invariant, nowadays named Estrada index, proposed by Estrada in 2000, is defined as [1]

$$
E E(G)=\sum_{i=1}^{n} e^{\lambda_{i}}
$$

Since then, the Estrada index has already found remarkable applications in biology, chemistry, and complex networks [2-5]. Some mathematical properties of the Estrada index, especially bounds for it have been established in [6-15]. For more results on the Estrada index, the readers are referred to recent papers [16-19].

Let $H=(V, \mathscr{E})$ be a simple and finite hypergraph with vertex set $V(G)=\left\{v_{1}, v_{2}, \ldots, v_{n}\right\}$ and hyperedge set $\mathscr{E}(G)=$ $\left\{E_{1}, E_{2}, \ldots, E_{m}\right\}$. The hypergraph $H$ is called linear if two hyperedges intersect in one vertex at most and also $h$-uniform if $\left|E_{i}\right|=h$ for each $E_{i}$ in $\mathscr{E}, i=1,2, \ldots, m$. An $h$-uniform hypertree is a connected linear $h$-hypergraph without cycles. An $h$-uniform linear hypertree is called 3 -uniform linear hypertree if $h$ is equal to 3 . Denoted by $\mathcal{S}_{m}^{h}$ an $h$-uniform linear star with $m$ hyperedges. More details on hypergraphs can be found in [20].

Let $A(H)$ denote a square symmetric matrix in which the diagonal elements $a_{i j}$ are zero, and other elements $a_{i j}$ represent the number of hyperedges containing both vertices $v_{i}$ and $v_{j}$ (for undirected hypergraphs, $a_{i j}=a_{j i}$ ). Let $\lambda_{1}$, $\lambda_{2}, \ldots, \lambda_{n}$ be the eigenvalues of $A(H)$ of $H$. The subhypergraph centrality of a hypergraph $H$, firstly put forward by Estrada and Rodríguez-Velázquez in 2006, is defined as [21]

$$
\left\langle C_{S H}\right\rangle=\frac{1}{n} \sum_{i=1}^{n} C_{S H}(i)=\frac{1}{n} \sum_{i=1}^{n} e^{\lambda_{i}} .
$$

They revealed that the subhypergraph centrality provides a measure of the centrality of complex hypernetworks (social, reaction, metabolic, protein, food web, etc). For convenience, we call the subhypergraph centrality of a hypergraph its Estrada index and define the Estrada index as

$$
E E(H)=\sum_{i=1}^{n} e^{\lambda_{i}} .
$$

Thus far, results on the Estrada index of hypergraph seem to be few although the Estrada index of graph has numerous applications. So our main goal is to investigate the Estrada index of 3-uniform linear hypertrees. In this paper, 


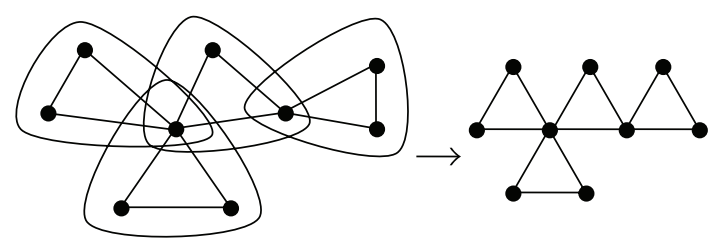

(a) 3-uniform linear hypertree $H \quad$ (b) Completely connected graph of $H$

FIGURE 1: A 3-uniform linear hypertree $H$ and its completely connected graph $G_{H}$.

we determine the unique 3-uniform linear hypertree with the maximum Estrada index among the set of 3-uniform linear hypertrees.

\section{Preliminaries}

For a hypergraph $H$ of order $n$, its completely connected graph, denoted by $G_{H}$, is a graph which has the same order and in which two vertices are adjacent if they share one hyperedge. Obviously, $G_{H}$ is a multigraph. For an $h$-uniform linear hypergraph $H, G_{H}$ is a simple graph. According to the definition of adjacency matrix of hypergraph, it is easy to see that both a 3-uniform linear hypertree $H$ and its completely connected graph $G_{H}$ have the same adjacency matrix; see Figure 1. Then, they have the identical Estrada index. Thus, we investigate the Estrada index of its completely connected graphs instead of the 3-uniform linear hypertrees in this paper.

We use $M_{k}(G)=\sum_{i=1}^{n} \lambda_{i}^{k}$ to denote the $k$ th spectral moment of the graph $G$. It is well-known [22] that $M_{k}(G)$ is equal to the number of closed walks of length $k$ in $G$. Obviously, for any graph $G, M_{0}(G)=n, M_{1}(G)=0, M_{2}(G)=$ $2 m, M_{3}(G)=6 t$, and $M_{4}(G)=2 \sum_{i=1}^{n} d_{i}^{2}-2 m+8 q$, where $t, q$, and $d_{i}=d_{G}\left(v_{i}\right)$ are the number of triangles, the number of quadrangles, and the degree of vertex $v_{i}$ in graph $G$, respectively. Then

$$
E E(G)=\sum_{i=1}^{n} \sum_{k=0}^{\infty} \frac{\lambda_{i}^{k}}{k !}=\sum_{k=0}^{\infty} \frac{M_{k}(G)}{k !}
$$

For $u, v \in V(G)$, denote by $\mathscr{W}_{k}(G ; u, v)$ the set of $(u, v)$-walks of length $k$ in $G$. Obviously, $M_{k}(G ; u, v)=$ $\left|\mathscr{W}_{k}(G ; u, v)\right|$. For convenience, let $\mathscr{W}_{k}(G ; u)=\mathscr{W}_{k}(G ; u, u)$ and $M_{k}(G ; u)=M_{k}(G ; u, u)$. Let $W$ be a $(u, v)$-walk in graph $G$; we denote by $W^{-1}$ a $(v, u)$-walk obtained from $W$ by reversing $W$.

For any two graphs $G_{1}$ and $G_{2}$, if $M_{k}\left(G_{1}\right) \geq M_{k}\left(G_{2}\right)$ for all integers $k>0$, then $E E\left(G_{1}\right) \geq E E\left(G_{2}\right)$. Moreover, if the strict inequality $M_{k}\left(G_{1}\right)>M_{k}\left(G_{2}\right)$ holds for at least one value $k>0$, then $E E\left(G_{1}\right)>E E\left(G_{2}\right)$.

Denote by $\Gamma(n, m)$ the set of connected graphs on $n$ vertices and $m$ triangles such that any two triangles have a common vertex at most. Apparently, for a 3-uniform linear hypertree $H$ on $n$ vertices and $m$ hyperedges, $G_{H} \in \Gamma(n, m)$. Now we study the Estrada index of a graph in $\Gamma(n, m)$.

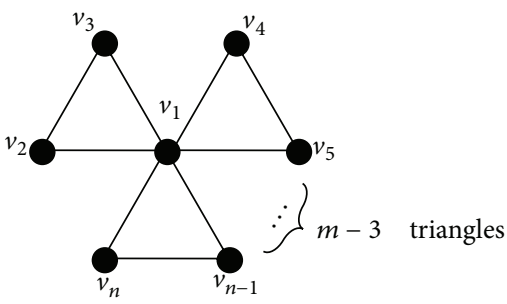

Figure 2: The star $S_{m}^{3}$ on $m$ triangles.

\section{Maximum Estrada Index of 3-Uniform Linear Hypertrees}

In this section, we determine the maximum value of Estrada index among the set of 3-uniform linear hypertrees.

Lemma 1. Let $S_{m}^{3}$ be star which is the completely connected graph of $S_{m}^{3}$ with $m$ hyperedges. It is easily found that the star $S_{m}^{3}$ has $n$ vertices labled $v_{1}, v_{2}, v_{3}, \ldots, v_{n}$ and $m=(n-1) / 2$ triangles. Let $k$ be a positive integer; then there is an injection $\xi$ from $\mathscr{W}_{k}\left(S_{m}^{3} ; v_{2}\right)$ to $\mathscr{W}_{k}\left(S_{m}^{3} ; v_{1}\right)$, and $\xi$ is not surjective for $n \geq 5,2 \leq m \leq(n-1) / 2$, and $k>1$, where $\mathscr{W}_{k}\left(S_{m}^{3} ; v_{2}\right)$ and $\mathscr{W}_{k}\left(S_{m}^{3} ; v_{1}\right)$ are the sets of closed walks of length $k$ of $v_{2}$ and $v_{1}$ in $S_{m}^{3}$, respectively; see Figure 2.

Proof. Firstly, we construct a mapping $\varphi$ from $\mathscr{W}_{k}\left(S_{m}^{3} ; v_{2}\right)$ to $\mathscr{W}_{k}\left(S_{m}^{3} ; v_{1}\right)$. For $W \in \mathscr{W}_{k}\left(S_{m}^{3} ; v_{2}\right)$, let $\varphi(W)$ be the closed walk obtained from $W$ by replacing $v_{1}$ by $v_{2}$ and $v_{2}$ by $v_{1}$. Obviously, $\varphi(W) \in \mathscr{W}_{k}\left(S_{m}^{3} ; v_{1}\right)$ and $\varphi$ is a bijection.

Secondly, we construct a mapping $\xi$ from $\mathscr{W}_{k}\left(S_{m}^{3} ; v_{2}\right)$ to $\mathscr{W}_{k}\left(S_{m}^{3} ; v_{1}\right)$. For $W \in \mathscr{W}_{k}\left(S_{m}^{3} ; v_{2}\right)$, we consider the following cases.

Case 1. Suppose $W$ does not pass the edge $v_{1} v_{t}$ for $t \geq 4$; then $\xi(W)=\varphi(W)$.

Case 2. Suppose $W$ passes the edge $v_{1} v_{t}$ for $t \geq 4$. For $W \in \mathscr{W}_{k}\left(S_{m}^{3} ; v_{2}\right)$, we may uniquely decompose $W$ into three sections $W_{1} W_{2} W_{3}$, where $W_{1}$ is the longest $\left(v_{2}, v_{1}\right)$-section of $W$ without $v_{t}, W_{2}$ is the internal longest $\left(v_{t}, v_{t}^{\prime}\right)$-section of $W$ for $t^{\prime} \geq 4$, and the last $W_{3}$ is the remaining $\left(v_{1}, v_{2}\right)$-section of $W$ not containing $v_{t}$. We consider the following three subcases. 


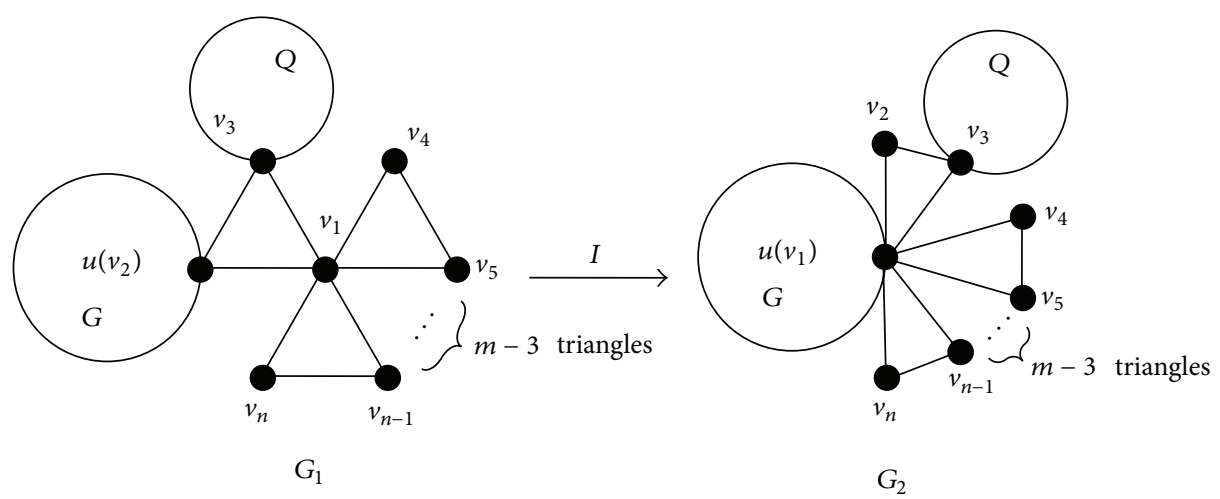

FIGURE 3: Transformation $I$.

Case 2.1. If both $W_{1}$ and $W_{3}$ contain the vertex $v_{3}$, we may uniquely decompose $W_{1}$ into two sections $W_{11} W_{12}$ and decompose $W_{3}$ into two sections $W_{31} W_{32}$, where $W_{11}$ is the shortest $\left(v_{2}, v_{3}\right)$-section of $W_{1}, W_{12}$ is the remaining $\left(v_{3}, v_{1}\right)$ section of $W_{1}, W_{31}$ is the longest $\left(v_{1}, v_{3}\right)$-section of $W_{3}$, and $W_{32}$ is the remaining $\left(v_{3}, v_{2}\right)$-section of $W_{3}$.

Let $\xi(W)=\xi\left(W_{11}\right) \xi\left(W_{12}\right) \xi\left(W_{2}\right) \xi\left(W_{31}\right) \xi\left(W_{32}\right)$, where $\xi\left(W_{12}\right)=W_{12}, \xi\left(W_{2}\right)=W_{2}, \xi\left(W_{31}\right)=W_{31}, \xi\left(W_{11}\right)$ is a $\left(v_{1}, v_{3}\right)$ walk obtained from $W_{11}$ replacing $v_{1}$ by $v_{2}$ and $v_{2}$ by $v_{1}$, and $\xi\left(W_{32}\right)$ is a $\left(v_{3}, v_{1}\right)$-walk obtained from $W_{32}$ replacing $v_{1}$ by $v_{2}$ and $v_{2}$ by $v_{1}$.

Case 2.2. If $W_{1}$ contains the vertex $v_{3}$ and $W_{3}$ does not contain $v_{3}$, let $\xi(W)=\xi\left(W_{1}\right) \xi\left(W_{2}\right) \xi\left(W_{3}\right)$, where $\xi\left(W_{2}\right)=W_{2}, \xi\left(W_{1}\right)$ is a $\left(v_{1}, v_{1}\right)$-walk obtained from $W_{1}$ replacing its first vertex $v_{2}$ by $v_{1} v_{2}$, and $\xi\left(W_{3}\right)$ is a $\left(v_{1}, v_{1}\right)$-walk obtained from $W_{3}$ replacing its last two vertices $v_{1} v_{2}$ by $v_{1}$.

Case 2.3. If $W_{1}$ does not contain the vertex $v_{3}$, let $\xi(W)=$ $\xi\left(W_{1}\right) \xi\left(W_{2}\right) \xi\left(W_{3}\right)$, where $\xi\left(W_{2}\right)=W_{2}, \xi\left(W_{1}\right)$ is a $\left(v_{1}, v_{1}\right)$-walk obtained from $W_{1}$ replacing its first two vertices $v_{2} v_{1}$ by $v_{1}$, and $\xi\left(W_{3}\right)$ is a $\left(v_{1}, v_{1}\right)$-walk obtained from $W_{3}$ replacing its last vertex $v_{2}$ by $v_{2} v_{1}$.

For example, in star $S_{3}^{3}$ on 7 vertices and 3 triangles, $W=$ $v_{2} v_{3} v_{1} v_{2} v_{1} v_{3} v_{2}$ is a closed walk of length 6 of $v_{2}$ not passing the edge $v_{1} v_{t}$. By Case 1 , we have

$$
\xi(W)=v_{1} v_{3} v_{2} v_{1} v_{2} v_{3} v_{1} .
$$

$W^{\prime}=v_{2} v_{3} v_{1} v_{4} v_{5} v_{1} v_{6} v_{7} v_{1} v_{2}$ is a closed walk of length 9 of $v_{2}$ passing the edge $v_{1} v_{t}$. By Case 2.2, we get

$$
\xi\left(W^{\prime}\right)=v_{1} v_{2} v_{3} v_{1} v_{4} v_{5} v_{1} v_{6} v_{7} v_{1}
$$

$W^{\prime \prime}=v_{2} v_{1} v_{2} v_{1} v_{4} v_{5} v_{1} v_{2} v_{3} v_{1} v_{6} v_{7} v_{1} v_{3} v_{2}$ is a closed walk of length 14 of $v_{2}$ passing the edge $v_{1} v_{t}$. By Case 2.3, we obtain

$$
\xi\left(W^{\prime \prime}\right)=v_{1} v_{2} v_{1} v_{4} v_{5} v_{1} v_{2} v_{3} v_{1} v_{6} v_{7} v_{1} v_{3} v_{2} v_{1}
$$

Obviously, $\xi(W) \in \mathscr{W}_{k}\left(S_{m}^{3} ; v_{1}\right), \xi$ is an injective and not a surjective for $n \geq 5$, and $k \geq 1$.

Lemma 2. Let $u$ be a nonisolated vertex of a connected graph G. If $G_{1}$ and $G_{2}$ are the graphs obtained from $G$ by identifying an external vertex $v_{2}$ and the center vertex $v_{1}$ of the union of $S_{m}^{3} \cup Q$ to $u$, respectively, where $\left|V\left(S_{m}^{3}\right)\right|=n$, Q is either empty graph or nonempty graph. Then $M_{k}\left(G_{1}\right)<M_{k}\left(G_{2}\right)$ for $n \geq 5$ and $k \geq 4$; see Figure 3.

Proof. Let $\mathscr{W}_{k}\left(G_{i}\right)\left(\mathscr{W}_{k}(G), \mathscr{W}_{k}\left(S_{m}^{3} \cup Q\right)\right.$, resp.) be the set of closed walks of length $k$ of $G_{i}\left(G, S_{m}^{3} \cup Q\right.$, resp.) for $i=1,2$. Then $\mathscr{W}_{k}\left(G_{i}\right)=\mathscr{W}_{k}(G) \cup \mathscr{W}_{k}\left(S_{m}^{3} \cup Q\right) \cup X_{i}$ is a partition, where $X_{i}$ is the set of closed walks of length $k$ of $G_{i}$; each of them contains both at least one edge in $E(G)$ and at least one edge in $E\left(S_{m}^{3} \cup Q\right)$. So $M_{k}\left(G_{i}\right)=\left|\mathscr{W}_{k}(G)\right|+\left|\mathscr{W}_{k}\left(S_{m}^{3} \cup Q\right)\right|+$ $\left|X_{i}\right|=M_{k}(G)+M_{k}\left(S_{m}^{3} \cup Q\right)+\left|X_{i}\right|$. Thus we need to show the inequality $\left|X_{1}\right|<\left|X_{2}\right|$.

We construct a mapping $\eta$ from $X_{1}$ to $X_{2}$ and consider the following four cases.

Case 1. Suppose $W$ is a closed walk starting from $u \in V(G)$ in $X_{1}$. For $W \in X_{1}$, let $\eta(W)=\left(W-W \cap\left(S_{m}^{3} \cup Q\right)\right) \cup \xi\left(W \cap\left(S_{m}^{3} \cup\right.\right.$ Q)); that is, $\eta(W)$ is the closed walk in $X_{2}$ obtained from $W$ by replacing its every section in $S_{m}^{3} \cup Q$ with its image under the map $\xi$.

Case 2. Suppose $W$ is a closed walk starting at $v_{1}$ in $X_{1}$. For $W \in X_{1}$, we may uniquely decompose $W$ into three sections $W_{1} W_{2} W_{3}$, where $W_{1}$ is the longest $\left(v_{1}, v_{2}\right)$-section of $W$ without vertices $u_{0}, \ldots, u_{t}^{\prime \prime} \in V(G), W_{2}$ is the internal longest $\left(u_{0}, u_{t}^{\prime \prime}\right)$-section of $W$ (for which the internal vertices are some possible vertices in $\left.V\left(G_{1}\right)\right)$, and $W_{3}$ is the remaining $\left(v_{2}, v_{1}\right)$-section of $W$. Let $\eta(W)=\eta\left(W_{1}\right) \eta\left(W_{2}\right) \eta\left(W_{3}\right)$, where $\eta\left(W_{1}\right)=W_{1}^{-1}, \eta\left(W_{3}\right)=W_{3}^{-1}$, and $\eta\left(W_{2}\right)=\left(W_{2}-W_{2} \cap\left(S_{m}^{3} \cup\right.\right.$ Q) $) \cup \xi\left(W_{2} \cap\left(S_{m}^{3} \cup Q\right)\right)$; that is, $\eta\left(W_{2}\right)$ is a $\left(u_{0}, u_{t}^{\prime \prime}\right)$-walk from $W_{2}$ by replacing its every section in $S_{m}^{3} \cup Q$ with its image under the $\operatorname{map} \xi$.

Case 3. Suppose $W$ is a closed walk starting from $v_{3}$ or $w \in$ $V(Q)$ in $X_{1}$. For $W \in X_{1}$, we may uniquely decompose $W$ into three sections $W_{1} W_{2} W_{3}$, where $W_{1}$ is the longest $\left(v_{3}, v_{2}\right)$ (or $\left.\left(w, v_{2}\right)\right)$-section of $W$ without vertices $u_{0}, \ldots, u_{t}^{\prime \prime}, W_{2}$ is the internal longest $\left(u_{0}, u_{t}^{\prime \prime}\right)$-section of $W$ (for which the internal vertices are some possible vertices in $\left.V\left(G_{1}\right)\right)$, and $W_{3}$ is the remaining $\left(v_{2}, v_{3}\right)$ (or $\left.\left(v_{2}, w\right)\right)$-section of $W$ without vertices $u_{0}, \ldots, u_{t}^{\prime \prime}$. We have three subcases. 
Case 3.1. If both $W_{1}$ and $W_{3}$ do not pass edge $v_{1} v_{t}$, let $\eta(W)=$ $\eta\left(W_{1}\right) \eta\left(W_{2}\right) \eta\left(W_{3}\right)$, where $\eta\left(W_{2}\right)=\left(W_{2}-W_{2} \cap\left(S_{m}^{3} \cup Q\right)\right) \cup$ $\xi\left(W_{2} \cap\left(S_{m}^{3} \cup Q\right)\right), \eta\left(W_{1}\right)$ is a $\left(v_{3}, v_{1}\right)\left(\right.$ or $\left.\left(w, v_{1}\right)\right)$-walk obtained from $W_{1}$ replacing $v_{1}$ by $v_{2}$ and $v_{2}$ by $v_{1}$, and $\eta\left(W_{3}\right)$ is a $\left(v_{1}, v_{3}\right)$ (or $\left.\left(v_{1}, w\right)\right)$-walk obtained from $W_{3}$ replacing $v_{1}$ by $v_{2}$ and $v_{2}$ by $v_{1}$.

Case 3.2. If both $W_{1}$ and $W_{3}$ pass edge $v_{1} v_{t}$, we may anew decompose $W$ into five sections $W_{1} W_{2} W_{3} W_{4} W_{5}$, where $W_{1}$ is the longest $\left(v_{3}, v_{t}\right)$ (or $\left.\left(w, v_{t}\right)\right)$-section of $W$ (which do not contain vertices $\left.u_{0}, \ldots, u_{t}^{\prime \prime}\right), W_{2}$ is the second $\left(v_{1}, v_{2}\right)$-section of $W$ (for which the internal vertices, if exist, are only possible $\left.v_{1}, v_{2}, v_{3}, w \in V(Q)\right)$, the third $W_{3}$ is the internal longest $\left(u_{0}, u_{t}^{\prime \prime}\right)$-section of $W$ (for which the internal vertices are some possible vertices in $V\left(G_{1}\right)$ ), the fourth $W_{4}$ is the longest $\left(v_{2}, v_{1}\right)$-section of $W$ (for which the internal vertices, if exist, are only possible $\left.v_{1}, v_{2}, v_{3}, w \in V(Q)\right)$, and the last $W_{5}$ is the remaining $\left(v_{t}^{\prime}, v_{3}\right)\left(\right.$ or $\left.\left(v_{t}, w\right)\right)$-section of $W$. We have three subsubcases.

Case 3.2.1. If both $W_{2}$ and $W_{4}$ contain the vertex $v_{3}$, we may uniquely decompose $W_{2}$ into two sections $W_{21} W_{22}$ and $W_{4}$ into two sections $W_{41} W_{42}$, where $W_{21}$ is the longest $\left(v_{1}, v_{3}\right)$ section of $W_{2}, W_{22}$ is the remaining shortest $\left(v_{3}, v_{2}\right)$ of $W_{2}, W_{41}$ is the shortest $\left(v_{2}, v_{3}\right)$-section of $W_{4}$, and $W_{42}$ is the remaining longest $\left(v_{3}, v_{1}\right)$-section of $W_{4}$.

Let $\eta(W)=\eta\left(W_{1}\right) \eta\left(W_{21}\right) \eta\left(W_{22}\right) \eta\left(W_{3}\right) \eta\left(W_{41}\right) \eta\left(W_{42}\right)$ $\eta\left(W_{5}\right)$, where $\eta\left(W_{1}\right)=W_{1}, \eta\left(W_{21}\right)=W_{21}, \eta\left(W_{3}\right)=\left(W_{3}-W_{3} \cap\right.$ $\left.\left(S_{m}^{3} \cup Q\right)\right) \cup \xi\left(W_{3} \cap\left(S_{m}^{3} \cup Q\right)\right), \eta\left(W_{42}\right)=W_{42} \eta\left(W_{5}\right)=W_{5}$, $\eta\left(W_{22}\right)$ is a $\left(v_{3}, v_{1}\right)$-walk obtained from $W_{22}$ replacing $v_{1}$ by $v_{2}$ and $v_{2}$ by $v_{1}$, and $\eta\left(W_{41}\right)$ is a $\left(v_{1}, v_{3}\right)$-walk obtained from $W_{41}$ replacing $v_{1}$ by $v_{2}$ and $v_{2}$ by $v_{1}$.

Case 3.2.2. If $W_{2}$ does not contain the vertex $v_{3}$, let $\eta(W)=$ $\eta\left(W_{1}\right) \eta\left(W_{2}\right) \eta\left(W_{3}\right) \eta\left(W_{4}\right) \eta\left(W_{5}\right)$, where $\eta\left(W_{1}\right)=W_{1}, \eta\left(W_{3}\right)=$ $\left(W_{3}-W_{3} \cap\left(S_{m}^{3} \cup Q\right)\right) \cup \xi\left(W_{3} \cap\left(S_{m}^{3} \cup Q\right)\right), \eta\left(W_{5}\right)=W_{5}$, $\eta\left(W_{2}\right)$ is a $\left(v_{1}, v_{1}\right)$-walk obtained from $W_{2}$ replacing its last two vertices $v_{1} v_{2}$ by $v_{1}$, and $\eta\left(W_{4}\right)$ is a $\left(v_{1}, v_{1}\right)$-walk obtained from $W_{4}$ replacing its first vertex $v_{2}$ by $v_{1} v_{2}$.

Case 3.2.3. If $W_{2}$ contains the vertex $v_{3}$ and $W_{4}$ does not contain vertex $v_{3}$, let $\eta(W)=\eta\left(W_{1}\right) \eta\left(W_{2}\right) \eta\left(W_{3}\right) \eta\left(W_{4}\right) \eta\left(W_{5}\right)$, where $\eta\left(W_{1}\right)=W_{1}, \eta\left(W_{3}\right)=\left(W_{3}-W_{3} \cap\left(S_{m}^{3} \cup Q\right)\right) \cup \xi\left(W_{3} \cap\right.$ $\left.\left(S_{m}^{3} \cup Q\right)\right), \eta\left(W_{5}\right)=W_{5}, \eta\left(W_{2}\right)$ is a $\left(v_{1}, v_{1}\right)$-walk obtained from $W_{2}$ replacing its last vertex $v_{2}$ by $v_{2} v_{1}$, and $\eta\left(W_{4}\right)$ is a $\left(v_{1}, v_{1}\right)$ walk obtained from $W_{4}$ replacing its first two vertices $v_{2} v_{1}$ by $v_{1}$.

Case 3.3. If $W_{1}$ passes edge $v_{1} v_{t}$ and $W_{3}$ does not pass edge $v_{1} v_{t}$, we may anew decompose $W$ into four sections $W_{1} W_{2} W_{3} W_{4}$, where $W_{1}$ is the longest $\left(v_{3}, v_{t}\right)$ (or $\left.\left(w, v_{t}\right)\right)$ section of $W$ (which do not contain vertices $\left.u_{0}, \ldots, u_{t}^{\prime \prime}\right), W_{2}$ is the second $\left(v_{1}, v_{2}\right)$-section of $W$ (for which the internal vertices, if exist, are only possible $\left.v_{1}, v_{2}, v_{3}, w \in V(Q)\right)$, the third $W_{3}$ is the internal longest $\left(u_{0}, u_{t}^{\prime \prime}\right)$-section of $W$ (for which the internal vertices are some possible vertices in $\left.V\left(G_{1}\right)\right)$, and the last $W_{4}$ is the longest $\left(v_{2}, v_{3}\right)\left(\right.$ or $\left.\left(v_{2}, w\right)\right)$ section of $W$ (for which the internal vertices, if exist, are only possible $\left.v_{1}, v_{2}, v_{3}, w \in V(Q)\right)$. We consider the following two subsubcases.

Case 3.3.1. If $W_{2}$ contains vertex $v_{3}$, we may uniquely decompose $W_{2}$ into two sections $W_{21} W_{22}$, where $W_{21}$ is the longest $\left(v_{1}, v_{3}\right)$-section of $W_{2}$ and $W_{22}$ is the remaining shortest $\left(v_{3}, v_{2}\right)$-section of $W_{2}$.

Let $\eta(W)=\eta\left(W_{1}\right) \eta\left(W_{21}\right) \eta\left(W_{22}\right) \eta\left(W_{3}\right) \eta\left(W_{4}\right)$, where $\eta\left(W_{1}\right)=W_{1}, \eta\left(W_{21}\right)=W_{21}, \eta\left(W_{3}\right)=\left(W_{3}-W_{3} \cap\left(S_{m}^{3} \cup\right.\right.$ Q) $) \cup \xi\left(W_{3} \cap\left(S_{m}^{3} \cup Q\right)\right), \eta\left(W_{22}\right)$ is a $\left(v_{3}, v_{1}\right)$-walk obtained from $W_{22}$ replacing $v_{1}$ by $v_{2}$ and $v_{2}$ by $v_{1}$, and $\eta\left(W_{4}\right)$ is a $\left(v_{1}, v_{3}\right)$ (or $\left.\left(v_{1}, w\right)\right)$-walk obtained from $W_{4}$ replacing $v_{1}$ by $v_{2}$ and $v_{2}$ by $v_{1}$.

Case 3.3.2. If $W_{2}$ does not contain vertex $v_{3}$, let $\eta(W)=\eta\left(W_{1}\right)$ $\eta\left(W_{2}\right) \eta\left(W_{3}\right) \eta\left(W_{4}\right)$, where $\eta\left(W_{1}\right)=W_{1}, \eta\left(W_{3}\right)=\left(W_{3}-W_{3} \cap\right.$ $\left.\left(S_{m}^{3} \cup Q\right)\right) \cup \xi\left(W_{3} \cap\left(S_{m}^{3} \cup Q\right)\right), \eta\left(W_{2}\right)$ is a $\left(v_{1}, v_{1}\right)$-walk obtained from $W_{2}$ replacing its last two vertices $v_{1} v_{2}$ by $v_{1}$, and $\eta\left(W_{4}\right)$ is a $\left(v_{1}, v_{3}\right)\left(\right.$ or $\left.\left(v_{1}, w\right)\right)$-walk obtained from $W_{4}$ replacing its first vertex $v_{2}$ by $v_{1} v_{2}$.

Case 3.4. If $W_{1}$ does not pass edge $v_{1} v_{t}$ and $W_{3}$ passes edge $v_{1} v_{t}$, we may anew decompose $W$ into four sections $W_{1} W_{2} W_{3} W_{4}$, where $W_{1}$ is the longest $\left(v_{3}, v_{2}\right)$ (or $\left.\left(w, v_{2}\right)\right)$ section of $W$ (which do not contain vertices $u_{0}, \ldots, u_{t}^{\prime \prime}$ and must contain vertex $v_{3}$ ), the second $W_{2}$ is the internal longest $\left(u_{0}, u_{t}^{\prime \prime}\right)$-section of $W$ (for which the internal vertices are some possible vertices in $\left.V\left(G_{1}\right)\right), W_{3}$ is the third $\left(v_{2}, v_{1}\right)$ section of $W$ (for which the internal vertices, if exist, are only possible $\left.v_{1}, v_{2}, v_{3}, w \in V(Q)\right)$, and the last $W_{4}$ is the longest $\left(v_{t}, v_{3}\right)\left(\right.$ or $\left.\left(v_{t}, w\right)\right)$-section of $W$. We have two subsubcases.

Case 3.4.1. If $W_{3}$ contains vertex $v_{3}$, we may uniquely decompose it into two sections $W_{31} W_{32}$, where $W_{31}$ is the shortest $\left(v_{2}, v_{3}\right)$-section of $W_{3}$ and $W_{32}$ is the remaining longest $\left(v_{3}, v_{1}\right)$-section of $W_{3}$.

Let $\eta(W)=\eta\left(W_{1}\right) \eta\left(W_{2}\right) \eta\left(W_{31}\right) \eta\left(W_{32}\right) \eta\left(W_{4}\right)$, where $\eta\left(W_{2}\right)=\left(W_{2}-W_{2} \cap\left(S_{m}^{3} \cup Q\right)\right) \cup \xi\left(W_{2} \cap\left(S_{m}^{3} \cup Q\right)\right), \eta\left(W_{32}\right)=W_{32}$, $\eta\left(W_{4}\right)=W_{4}, \eta\left(W_{1}\right)$ is a $\left(v_{3}, v_{1}\right)$ (or $\left.\left(w, v_{1}\right)\right)$-walk obtained from $W_{1}$ replacing $v_{1}$ by $v_{2}$ and $v_{2}$ by $v_{1}$, and $\eta\left(W_{31}\right)$ is a $\left(v_{1}, v_{3}\right)$-walk obtained from $W_{31}$ replacing $v_{1}$ by $v_{2}$ and $v_{2}$ by $v_{1}$.

Case 3.4.2. If $W_{3}$ does not contain vertex $v_{3}$, let $\eta(W)=\eta\left(W_{1}\right)$ $\eta\left(W_{2}\right) \eta\left(W_{3}\right) \eta\left(W_{4}\right)$, where $\eta\left(W_{2}\right)=\left(W_{2}-W_{2} \cap S_{m}^{3}\right) \cup \xi\left(W_{2} \cap S_{m}^{3}\right)$, $\eta\left(W_{4}\right)=W_{4}, \eta\left(W_{1}\right)$ is a $\left(v_{3}, v_{1}\right)\left(\right.$ or $\left.\left(w, v_{1}\right)\right)$-walk obtained from $W_{1}$ replacing its last vertex $v_{2}$ by $v_{2} v_{1}$, and $\eta\left(W_{3}\right)$ is a $\left(v_{1}, v_{1}\right)$-walk obtained from $W_{3}$ replacing its first two vertices $v_{2} v_{1}$ by $v_{1}$.

Case 4. Suppose $W$ is a closed walk starting from $v_{i}$ for $i=4,5,6, \ldots, n$ in $X_{1}$. For $W \in X_{1}$, we may uniquely decompose $W$ into five sections $W_{1} W_{2} W_{3} W_{4} W_{5}$, where $W_{1}$ is the longest $\left(v_{i}, v_{t}\right)$-section of $W$ (which do not contain vertices $\left.u_{0}, \ldots, u_{t}^{\prime \prime}\right), W_{2}$ is the second $\left(v_{1}, v_{2}\right)$-section of $W$ (for which the internal vertices, if exist, are only possible $\left.v_{1}, v_{2}, v_{3}, w \in V(G)\right)$, the third $W_{3}$ is the internal longest $\left(u_{0}, u_{t}^{\prime \prime}\right)$-section of $W$ (for which the internal vertices are some possible vertices in $V\left(G_{1}\right)$ ), the fourth $W_{4}$ is the longest 


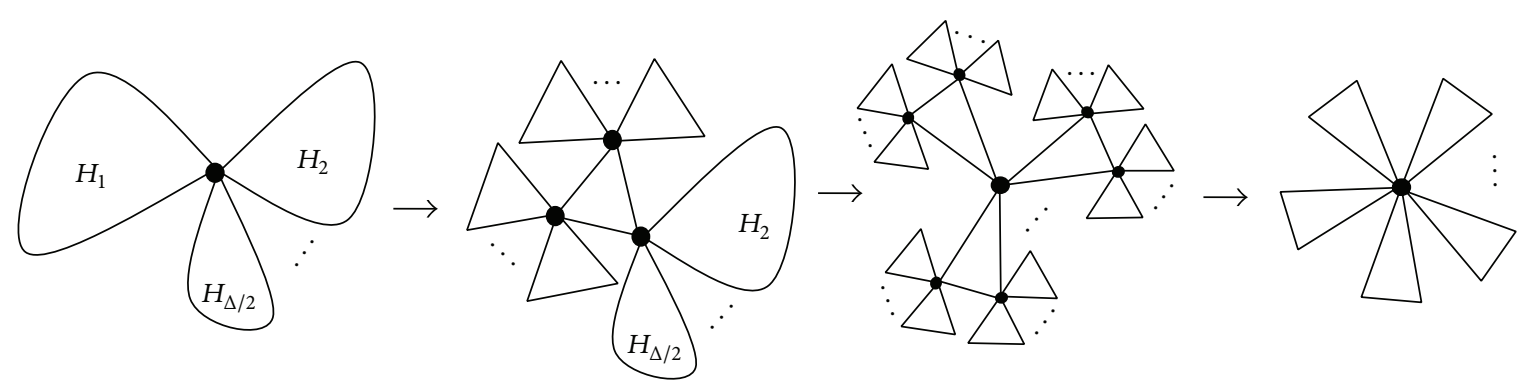

FIgURE 4: The procedure of transformation.

$\left(v_{2}, v_{1}\right)$-section of $W$ (for which the internal vertices, if exist, are only possible $\left.v_{1}, v_{2}, v_{3}, w \in V(G)\right)$, and the last $W_{5}$ is the remaining $\left(v_{t}^{\prime}, v_{i}\right)$-section of $W$. We have four subcases.

Case 4.1. If both $W_{2}$ and $W_{4}$ contain the vertex $v_{3}$, we may uniquely decompose $W_{2}$ into two sections $W_{21} W_{22}$ and decompose $W_{4}$ into two sections $W_{41} W_{42}$, where $W_{21}$ is the longest $\left(v_{1}, v_{3}\right)$-section of $W_{2}, W_{22}$ is the remaining shortest $\left(v_{3}, v_{2}\right)$ of $W_{2}, W_{41}$ is the shortest $\left(v_{2}, v_{3}\right)$-section of $W_{4}$, and $W_{42}$ is the remaining longest $\left(v_{3}, v_{1}\right)$-section of $W_{4}$.

Let $\eta(W)=\eta\left(W_{1}\right) \eta\left(W_{21}\right) \eta\left(W_{22}\right) \eta\left(W_{3}\right) \eta\left(W_{41}\right) \eta\left(W_{42}\right)$ $\eta\left(W_{5}\right)$, where $\eta\left(W_{1}\right)=W_{1}, \eta\left(W_{21}\right)=W_{21}, \eta\left(W_{3}\right)=\left(W_{3}-W_{3} \cap\right.$ $\left.\left(S_{m}^{3} \cup Q\right)\right) \cup \xi\left(W_{3} \cap\left(S_{m}^{3} \cup Q\right)\right), \eta\left(W_{42}\right)=W_{42} \eta\left(W_{5}\right)=W_{5}$, $\eta\left(W_{22}\right)$ is a $\left(v_{3}, v_{1}\right)$-walk obtained from $W_{22}$ replacing $v_{1}$ by $v_{2}$ and $v_{2}$ by $v_{1}$, and $\eta\left(W_{41}\right)$ is a $\left(v_{1}, v_{3}\right)$-walk obtained from $W_{41}$ replacing $v_{1}$ by $v_{2}$ and $v_{2}$ by $v_{1}$.

Case 4.2. If $W_{2}$ contains the vertex $v_{3}$ and $W_{4}$ does not contain vertex $v_{3}$, let $\eta(W)=\eta\left(W_{1}\right) \eta\left(W_{2}\right) \eta\left(W_{3}\right) \eta\left(W_{4}\right) \eta\left(W_{5}\right)$, where $\eta\left(W_{1}\right)=W_{1}, \eta\left(W_{3}\right)=\left(W_{3}-W_{3} \cap\left(S_{m}^{3} \cup Q\right)\right) \cup \xi\left(W_{3} \cap\left(S_{m}^{3} \cup\right.\right.$ Q)), $\eta\left(W_{5}\right)=W_{5}, \eta\left(W_{2}\right)$ is a $\left(v_{1}, v_{1}\right)$-walk obtained from $W_{2}$ replacing its last vertex $v_{2}$ by $v_{2} v_{1}$, and $\eta\left(W_{4}\right)$ is a $\left(v_{1}, v_{1}\right)$-walk obtained from $W_{4}$ replacing its first two vertices $v_{2} v_{1}$ by $v_{1}$.

Case 4.3. If $W_{2}$ does not contain the vertex $v_{3}$, let $\eta(W)=$ $\eta\left(W_{1}\right) \eta\left(W_{2}\right) \eta\left(W_{3}\right) \eta\left(W_{4}\right) \eta\left(W_{5}\right)$, where $\eta\left(W_{1}\right)=W_{1}, \eta\left(W_{3}\right)=$ $\left(W_{3}-W_{3} \cap\left(S_{m}^{3} \cup Q\right)\right) \cup \xi\left(W_{3} \cap\left(S_{m}^{3} \cup Q\right)\right), \eta\left(W_{5}\right)=W_{5}, \eta\left(W_{2}\right)$ is a $\left(v_{1}, v_{1}\right)$-walk obtained from $W_{2}$ by replacing its last two vertices $v_{1} v_{2}$ by $v_{1}$, and $\eta\left(W_{4}\right)$ is a $\left(v_{1}, v_{1}\right)$-walk obtained from $W_{4}$ by replacing its first vertex $v_{2}$ by $v_{1} v_{2}$.

For example,

$$
\begin{aligned}
& \eta_{1}\left(u_{0} u_{1} \cdots u_{r} v_{2} v_{3} w_{1} \cdots w_{t} v_{3} v_{1} v_{2} u_{1}^{\prime} \cdots\right. \\
& \left.u_{s}^{\prime} v_{2} v_{1} v_{3} v_{2} u_{1}^{\prime \prime} \cdots u_{t}^{\prime \prime} u_{0}\right) \\
& =u_{0} u_{1} \cdots u_{r} v_{1} v_{3} w_{1} \cdots w_{t} v_{3} v_{2} v_{1} u_{1}^{\prime} \cdots \\
& u_{s}^{\prime} v_{1} v_{2} v_{3} v_{1} u_{1}^{\prime \prime} \cdots u_{t}^{\prime \prime} u_{0}, \\
& \eta_{1}\left(u_{0} u_{1} \cdots u_{r} v_{2} v_{3} w_{1} \cdots w_{t} v_{3} v_{1} v_{4} v_{5} v_{1} v_{2} u_{1}^{\prime} \cdots\right. \\
& \left.u_{s}^{\prime} v_{2} v_{3} v_{5} v_{4} v_{1} v_{3} v_{2} u_{1}^{\prime \prime} \cdots u_{t}^{\prime \prime} u_{0}\right) \\
& =u_{0} u_{1} \cdots u_{r} v_{1} v_{2} v_{3} w_{1} \cdots w_{t} v_{3} v_{1} v_{4} v_{5} v_{1} u_{1}^{\prime} \\
& \cdots u_{s}^{\prime} v_{1} v_{3} v_{5} v_{4} v_{1} v_{3} v_{1} u_{1}^{\prime \prime} \cdots u_{t}^{\prime \prime} u_{0},
\end{aligned}
$$

$$
\begin{aligned}
& \eta_{1}\left(v_{3} w_{1} \cdots w_{t} v_{3} v_{2} u_{1} \cdots u_{r} v_{2} v_{1} v_{5} v_{4} v_{1} v_{2} u_{1}^{\prime} \cdots u_{s}^{\prime} v_{2} v_{1} v_{3}\right) \\
& =v_{3} w_{1} \cdots w_{t} v_{3} v_{1} u_{1} \cdots u_{r} v_{1} v_{5} v_{4} v_{1} v_{2} v_{1} u_{1}^{\prime} \cdots u_{s}^{\prime} v_{1} v_{2} v_{3}, \\
& \eta_{1}\left(v_{3} v_{2} v_{1} v_{7} v_{6} v_{1} v_{2} v_{1} v_{2} u_{1} \cdots u_{r} v_{2} v_{1} v_{4} v_{5} v_{1} v_{3} v_{2} u_{1}^{\prime} \cdots\right. \\
& \left.u_{s}^{\prime} v_{2} v_{1} v_{7} v_{6} v_{1} v_{2} v_{3}\right) \\
& =v_{3} v_{2} v_{1} v_{7} v_{6} v_{1} v_{2} v_{1} u_{1} \cdots u_{r} v_{1} v_{4} v_{5} v_{1} v_{3} v_{2} v_{1} u_{1}^{\prime} \\
& \cdots u_{s}^{\prime} v_{1} v_{2} v_{1} v_{7} v_{6} v_{1} v_{2} v_{3}, \\
& \eta_{1}\left(v_{1} v_{4} v_{5} v_{1} v_{3} v_{2} u_{1} \cdots u_{r} v_{2} v_{3} v_{2} v_{1} v_{4} v_{5} v_{1} v_{3} v_{2} u_{1}^{\prime} \cdots u_{s}^{\prime} v_{2} v_{1}\right) \\
& =v_{2} v_{3} v_{1} v_{5} v_{4} v_{1} u_{1} \cdots u_{r} v_{1} v_{3} v_{2} v_{1} v_{4} v_{5} v_{1} v_{3} v_{1} u_{1}^{\prime} \cdots u_{s}^{\prime} v_{1} v_{2}, \\
& \eta_{1}\left(v_{4} v_{5} v_{1} v_{3} v_{2} u_{1} \cdots u_{r} v_{2} v_{3} v_{2} v_{1} v_{4} v_{5} v_{1} v_{3} v_{2} u_{1}^{\prime}\right. \\
& \left.\quad \cdots u_{s}^{\prime} v_{2} v_{3} v_{2} v_{1} v_{5} v_{4}\right) \\
& =v_{4} v_{5} v_{1} v_{3} v_{1} u_{1} \cdots u_{r} v_{1} v_{3} v_{2} v_{1} v_{4} v_{5} v_{1} v_{3} v_{1} u_{1}^{\prime} \\
& \cdots u_{s}^{\prime} v_{1} v_{3} v_{2} v_{1} v_{5} v_{4},
\end{aligned}
$$

where $u_{0}, u_{1}, \ldots, u_{r}, u_{1}^{\prime}, \ldots, u_{s}^{\prime}, u_{1}^{\prime \prime}, \ldots, u_{t}^{\prime \prime}$ are vertices in $G$ and $w_{1}, \ldots, w_{t}$ are vertices in $Q$.

By Lemma $1, \xi$ is injective and not surjective. It is easily shown that $\eta$ is also injective and not surjective. Thus $\left|X_{1}\right|<$ $\left|X_{2}\right|, M_{k}\left(G_{1}\right)<M_{k}\left(G_{2}\right)$.

Theorem 3. Let $G_{H}$ be an arbitrary graph on $n$ vertices in set $\Gamma(n, m)$, where $n>5$. Then $E E\left(G_{H}\right) \leq E E\left(S_{m}^{3}\right)$ with the equality holding if and only if $G_{H} \cong S_{m}^{3}$.

Proof. Determine a vertex $v$ of the maximum degree $\Delta$ as a root in $G_{H}$, and let $k \geq 4$ be an integer. Let $G_{H_{i}}$ be the completely connected graph of 3-uniform linear hypertree $H_{i}$ attached at $v$, and let $m_{i}$ be the number of triangles of $G_{H_{i}}$ for $i=1,2, \ldots, \Delta / 2$, respectively. We can repeatedly apply this transformation from Lemma 2 at some vertices whose degrees are not equal to two or $2 m_{i}$ in $G_{H_{i}}$ till $G_{H_{i}}$ becomes a star. From Lemma 2, it satisfies that each application of this transformation strictly increases the number of closed walks and also increases Estrada index. 
When all $G_{H_{i}}$ turn into stars, we can again use Lemma 2 at the vertex $v$ as long as there exists at least one vertex whose degree is not equal to two or $2 \sum m_{i}$, further increasing the number of closed walks. In the end of this procedure, we get the star $S_{m}^{3}$. The whole procedure of transformation is shown in Figure 4.

Lemma 4 (see [20]). Let $v$ be a vertex of a graph $G, G-\{v\}=$ $G-v$ for $v \in V(G)$, and $\mathscr{C}(v)$ the set of cycles containing $v$. Consider

$$
\begin{aligned}
P(G, \lambda)= & \lambda \cdot P(G-v, \lambda)-\sum_{v w \in E(G)} P(G-v-w, \lambda) \\
& -2 \sum_{Z \in \mathscr{C}(v)} P(G-V(Z), \lambda),
\end{aligned}
$$

where $P(G-v-w, \lambda)=1$ if $G$ is a single edge and $P(G-$ $V(Z), \lambda)=1$ if $G$ is a cycle.

Now, we calculate $E E\left(S_{m}^{3}\right)$. Applying Lemma 4, we have

$$
P\left(S_{m}^{3}, \lambda\right)=(\lambda+1)^{(n-1) / 2}(\lambda-1)^{(n-3) / 2}\left(\lambda^{2}-\lambda-n+1\right) .
$$

By some simple calculating, we achieve the following eigenvalues:

$$
\begin{gathered}
\lambda_{1}=\lambda_{2}=\cdots=\lambda_{(n-1) / 2}=-1, \\
\lambda_{(n+1) / 2}=\lambda_{(n+3) / 2}=\cdots=\lambda_{n-2}=1, \\
\lambda_{n-1}=\frac{1-\sqrt{4 n-3}}{2}, \quad \lambda_{n}=\frac{1+\sqrt{4 n-3}}{2} .
\end{gathered}
$$

Then, we obtain

$$
\begin{aligned}
E E\left(S_{m}^{3}\right)= & \frac{(n-1)}{2 e}+\frac{(n-3)}{2} e \\
& +e^{(1+\sqrt{4 n-3}) / 2}+e^{(1-\sqrt{4 n-3}) / 2} .
\end{aligned}
$$

Theorem 3 shows that the star $S_{m}^{3}$ has the maximum Estrada index in set $\Gamma(n, m)$. Thus, according to previous definition, it is easy to show that the 3-uniform star $\delta_{m}^{3}$ has the maximum Estrada index among the set of 3-uniform linear hypertrees; that is,

$$
E E(H) \leq E E\left(\delta_{m}^{3}\right)
$$

where

$$
\begin{aligned}
E E\left(\mathcal{S}_{m}^{3}\right)= & \frac{(n-1)}{2 e}+\frac{(n-3)}{2} e \\
& +e^{(1+\sqrt{4 n-3}) / 2}+e^{(1-\sqrt{4 n-3}) / 2}
\end{aligned}
$$

\section{Conflict of Interests}

The authors declare that there is no conflict of interests regarding the publication of this paper.

\section{Acknowledgments}

This work was supported by Program for Changjiang Scholars and Innovative Research Team in University (PCSIRT)(no. ITR1068), Special 973 Program for Key Basic Research of the Ministry of Science and Technology, China (no. 2010CB334708), the National Natural Science Foundation of China (NSFC)(no. 60863006), Scientific Research Foundation of the Department of Science and Technology, Qinghai Province, China (no. 2012-Z-943).

\section{References}

[1] E. Estrada, "Characterization of 3D molecular structure," Chemical Physics Letters, vol. 319, no. 5-6, pp. 713-718, 2000.

[2] E. Estrada, "Characterization of the folding degree of proteins," Bioinformatics, vol. 18, no. 5, pp. 697-704, 2002.

[3] E. Estrada, "Characterization of the amino acid c ontration to the folding degree of proteins," Proteins, vol. 54, no. 4, pp. 727737, 2004.

[4] E. Estrada and J. A. Rodríguez-Velázquez, "Subgraph centrality in complex networks," Physical Review E: Statistical, Nonlinear, and Soft Matter Physics, vol. 71, no. 5, 9 pages, 2005.

[5] E. Estrada, J. A. Rodríguez-Velázquez, and M. Randić, "Atomic branching in molecules," International Journal of Quantum Chemistry, vol. 106, no. 4, pp. 823-832, 2006.

[6] K. C. Das and S. Lee, "On the Estrada index conjecture," Linear Algebra and its Applications, vol. 431, no. 8, pp. 1351-1359, 2009.

[7] H. Deng, "A proof of a conjecture on the Estrada index," MATCH, Communications in Mathematical and in Computer Chemistry, vol. 62, no. 3, pp. 599-606, 2009.

[8] Z. Du and B. Zhou, “The Estrada index of trees," Linear Algebra and Its Applications, vol. 435, no. 10, pp. 2462-2467, 2011.

[9] Z. Du and B. Zhou, "The Estrada index of unicyclic graphs," Linear Algebra and Its Applications, vol. 436, no. 9, pp. 3149-3159, 2012.

[10] Q. Deng and H. Chen, "On extremal bipartite unicyclic graphs," Linear Algebra and Its Applications, vol. 444, pp. 89-99, 2014.

[11] A. Ilić and D. Stevanović, “The Estrada index of chemical trees," Journal of Mathematical Chemistry, vol. 47, no. 1, pp. 305-314, 2010.

[12] W. Wang and W. Xu, "Graphs with the maximal Estrada indices," Linear Algebra and its Applications, vol. 446, pp. 314-328, 2014.

[13] H. Zhao and Y. Jia, "On the Estrada index of bipartite graph," MATCH: Communications in Mathematical and in Computer Chemistry, vol. 61, no. 2, pp. 495-501, 2009.

[14] B. Zhou and Z. Du, "Some lower bounds for Estrada index," Iranian Journal of Mathematical Chemistry, vol. 1, no. 2, pp. 6772, 2010.

[15] Z. Zhu, L. Tan, and Z. Qiu, "Tricyclic graph with maximal Estrada index," Discrete Applied Mathematics, vol. 162, pp. 364372, 2014.

[16] Z. Du, "An edge grafting theorem on the Estrada index of graphs and its applications," Discrete Applied Mathematics, vol. 161, no. 1-2, pp. 134-139, 2013.

[17] J. A. de la Peña, I. Gutman, and J. Rada, "Estimating the Estrada index," Linear Algebra and its Applications, vol. 427, no. 1, pp. 70-76, 2007. 
[18] I. Gutman, H. Deng, and S. Radenković, "The Estrada index," in Applications of Graph Spectra, D. Cvetković and I. Gutman, Eds., pp. 155-174, Mathematics Institute, Beograd, Serbia, 2011.

[19] J. Liu, X. Pan, and J. Cao, "Some properties on Estrada index of folded hypercubes networks," Abstract and Applied Analysis, vol. 2014, Article ID 167623, 6 pages, 2014.

[20] C. Berge, Graphs and Hypergraphs, Elsevier, New York, NY, USA, 1973.

[21] E. Estrada and J. A. Rodríguez-Velázquez, "Subgraph centrality and clustering in complex hyper-networks," Physica A: Statistical Mechanics and Its Applications, vol. 364, pp. 581-594, 2006.

[22] D. Cvetkoic, M. Doob, and H. Sachs, Spectra of Graphs. Theory and Application, vol. 87 of Pure and Applied Mathematics, Academic Press, New York, NY, USA, 1980. 


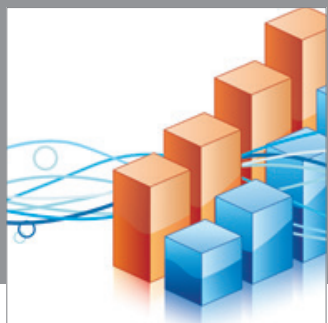

Advances in

Operations Research

mansans

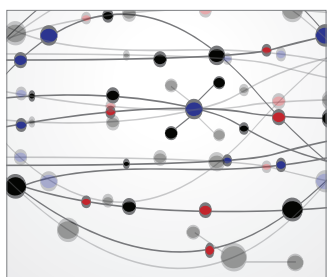

The Scientific World Journal
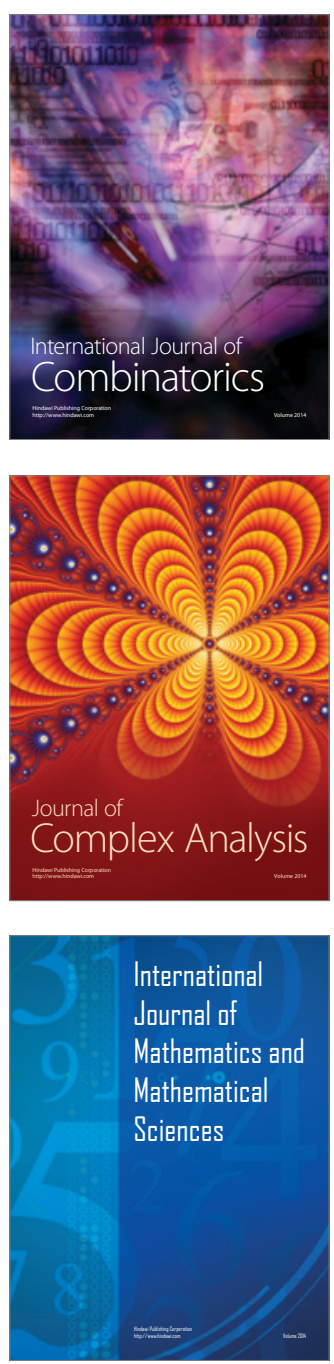
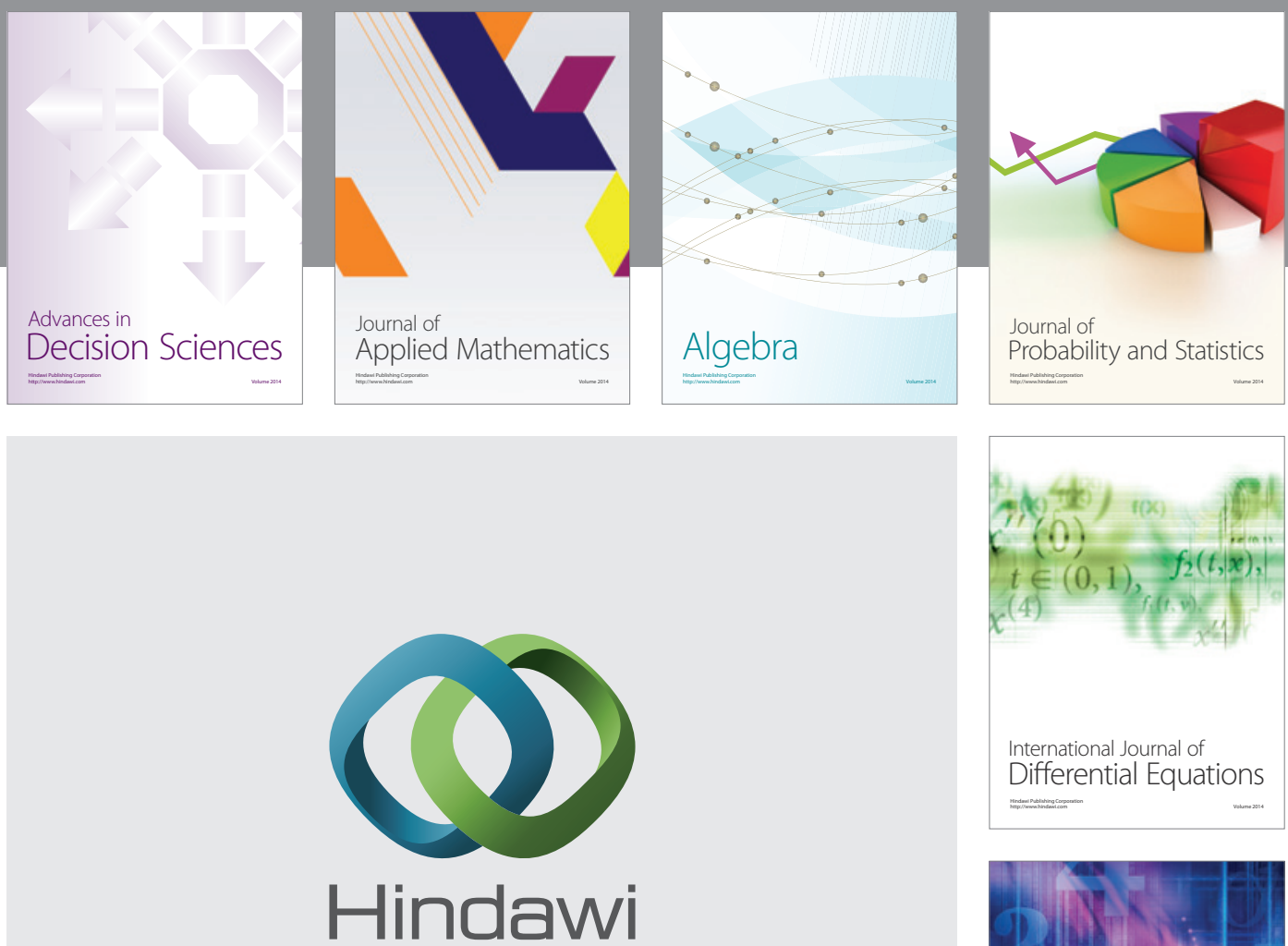

Submit your manuscripts at http://www.hindawi.com
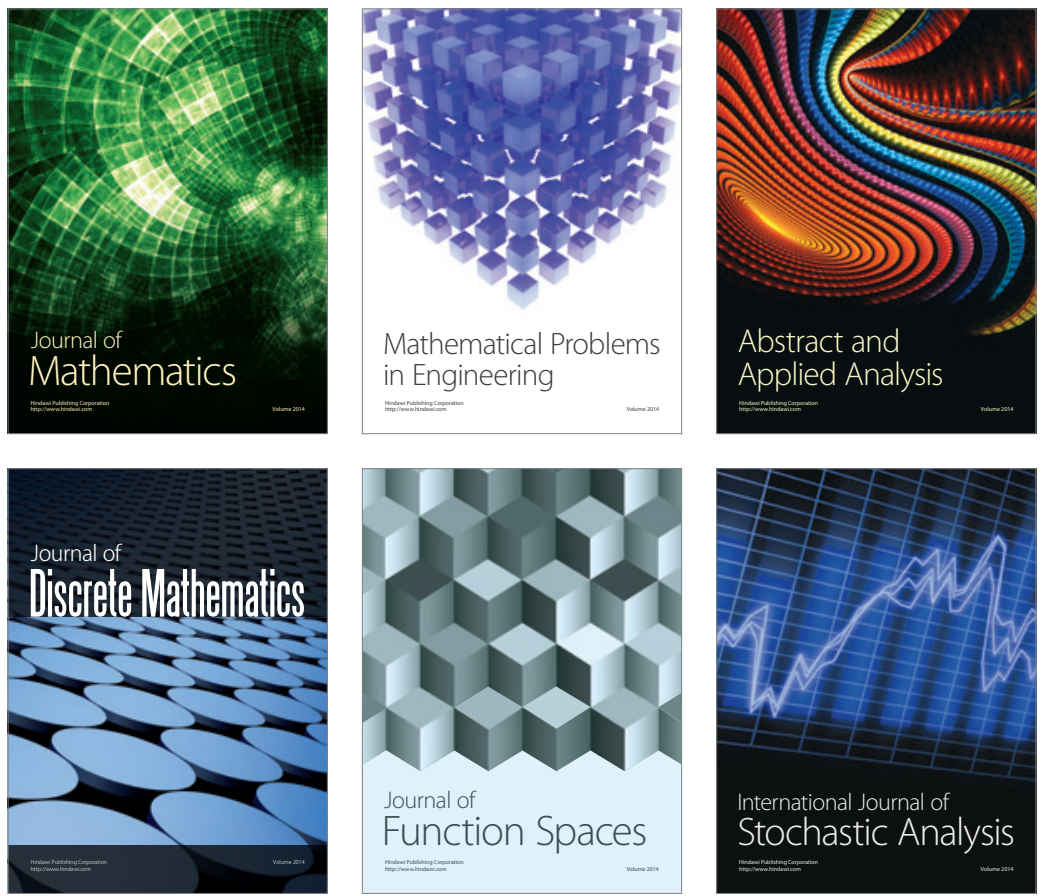

Journal of

Function Spaces

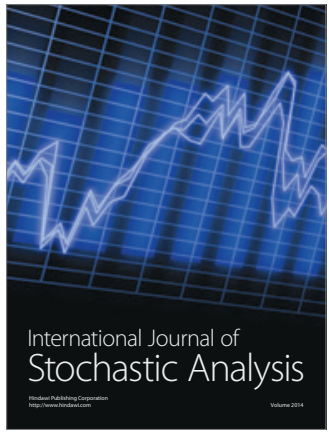

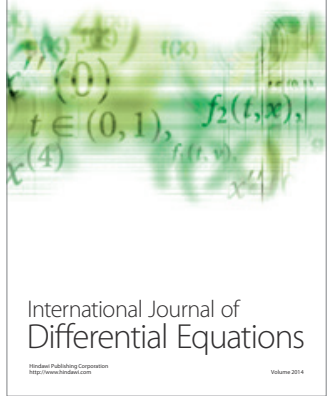
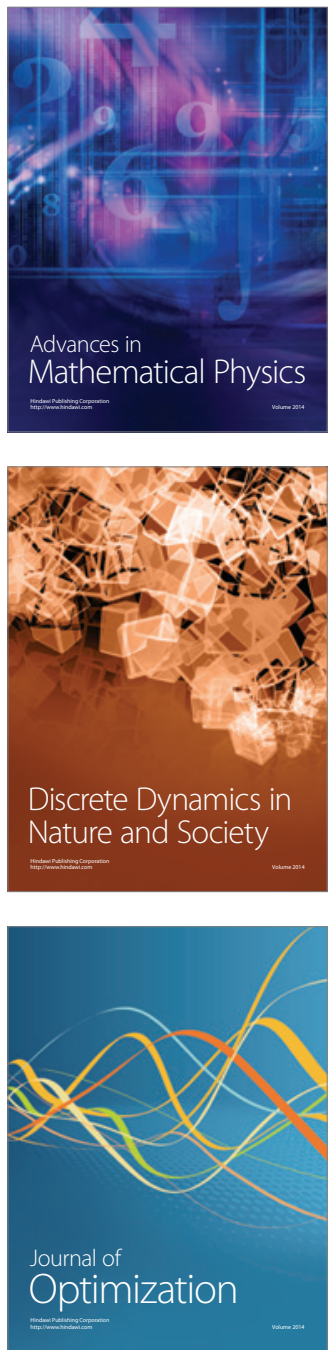